\title{
Efficacité du module des activités ludiques basées sur les stratégies de l'apprentissage coopératif pour le développement des compétences langagières chez les élèves des écoles préparatoires en FLE
}

\author{
Préparée par \\ Nadia Ahmed Maati Maati*
}

\section{Introduction}

Dans cette étude, on manipule deux volets principaux: dans un premier temps, le module des activités ludiques et son rôle dans le développement des compétences langagières chez les élèves du cycle préparatoire, on essaye de définir les deux concepts : " jeu " et "activité ludique ". On montre les différents types d'activités ludiques et leur utilité dans l'enseignement du FLE et son influence de développement des compétences langagières, dans un deuxième temps

\section{Cadre théorique:}

A. Les activités ludiques:

Dans l'enseignement/ apprentissage scolaire, les jeux font partie de la journée scolaire. Ils nous permettent à un temps de sortir de la monotonie de la classe, expérimenter, chanter, créer, imaginer et résoudre des problèmes. Parce que jouer, c'est vivre dans un autre monde qui rend l'élève très content, il génère de l'énergie et l'activité, cela rend l'élève inventé plus. Autrement dit, les jeux poussent l'élève à créer des choses nouvelles par une grande motivation

Selon le petit Robert, (1991 : p1046) : le « jeu » vient du mot latin « jocus » qui signifie « badinage, plaisanterie » ou en latin plus courant « amusement, divertissement ». Quant au mot « ludique »; c'est un dérivé du mot latin « lu dus » qui signifie relatif au jeu .

*Étude présentée pour compléter les exigences de l'obtention du grade de magistère en pédagogie Sous la direction de:

Prof. Dr. Adel Tawfik Mohamed, Professeur en didactique de FLE et doyen de la faculté de Pédagogie Université de la cité Sadate

Dr. Cherifa Abdallah Ahmed, Maître de conférences de FLE Faculté de Pédagogie Université du Canal de Suez 


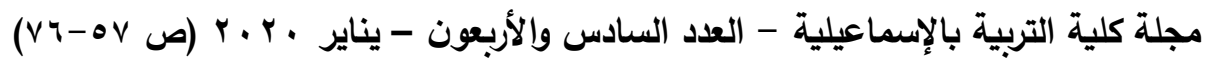

Selon le dictionnaire de français LAROUSSE: Jeu: non masculin (latin, plaisanterie) est défini comme: une activité d'ordre physique ou mental, non imposé, ne visant à aucune fin utilitaire, et à laquelle on s'adonne pour se divertir, en tirer un plaisir; participer à un jeu.

Selon le dictionnaire didactique : « le jeu en didactique des langues, un événement de communication interactive à deux ou plusieurs participant pour développer une compétence

L'objectif principal de l'enseignement en FLE est d'enseigner aux élèves à acquérir ou développer des compétences ou à communiquer par plusieurs moyens parmi lesquels la créativité et la découverte en même temps le plaisir d'apprendre en coopération avec les autres élèves dans la salle de classe.

L'objectif de l'apprentissage du FLE n'acquiert pas des connaissances académiques, mais utilise les connaissances dans la pratique de la vie quotidienne, comprendre et répondre à ce qui est dit et de s'exprimer afin de développer la performance langagière en FLE soit à l'oral ou à l'écrit

Selon Boudjema (2016), l'utilisation du jeu est une nécessité dans une classe d'une langue étrangère. Cette dernière incluse dans son acte didactique une pédagogie d'enseignement/ apprentissage qui emploie le jeu comme moyen, pour bénéficier de l'envie inévitable de l'enfant à jouer. D'une manière pédagogiquement étudiée et en tant qu'activité sérieuse sous une forme ludique, l'enseignant de la langue étrangère et précisément la langue française vont installer chez ses apprenants une compétence langagière par le billet du «jeu».

Dans ce sens affirmé Boadiwaa (2015): « les jeux pédagogiques comme un ensemble d'activités plaisantes ; amusantes et récréatives entreprises par un enseignant et ses élèves dans une classe de langue pour faciliter et promouvoir l'enseignement/ apprentissage de cette langue.»

Ainsi Freud (2010) explique que : «Chaque enfant qui joue se comporte comme un poète, dans la mesure où il se crée un monde propre, ou pour parler plus exactement, il arrange les choses de son monde suivant un ordre nouveau, à sa convenance ». 
On n'entende par activité ludique une activité d'amusante que détente telle que les jeux éducatifs, les chansons. Notons que ce n'est pas l'activité qui est ludique mais l'attitude et le déroulement qui rend l'activité ludique.

Selon Jean-Pierre Cuq (2003):« une activité d'apprentissage dite ludique est guidée par des règles de jeux et pratiqué pour le plaisir quelle procure, elle permet une communication entre apprenants en collecte d'informations, problème créativité, prise de décision. Orientée vers un objectif d'apprentissage, elle permet aux apprenants d'utilisée pour faire façon collaborative et créative l'ensemble de leurs ressources verbale et communicative.

C'est-à-dire que les activités ludiques sont des activités qui se font se forment des jeux et qui permettent aux élèves de communiquer et discuter entre eux en utilisant la langue.

Autrement dit le jeu pédagogique ne cherche pas le plaisir mais il cherche à faire apprendre des connaissances par d'autres

Selon Soltani Habiba (2017): «Dans le jeu pédagogique, le facteur du plaisir est peu, il n'est plus intrinsèquement lié à l'acte du jeu, cependant, cette sensation de plaisir émane de la vérification des connaissances par l'apprenant de sorte que le plaisir devient travail.»

Il est indispensable de citer ici que les jeux pédagogiques contribuent à l'acquisition de connaissances ou de compétences. Ils sont de plus en plus reconnus par les pédagogues et les enseignants comme utiles, pour les raisons suivantes:

- Les enfants aiment et ont besoin de jouer. Un contenu perçu comme ennuyeux, peut les passionner une fois formulé sous forme ludique.

- Les joueurs jouent ensemble : leur attention est soutenue, au contraire d'un cours où les élèves peuvent penser à autre chose.

- La motivation de gagner renforce l'attention

- Les jeux de société socialisent les enfants qui apprennent à respecter une règle commune, ce qui fait partie des objectifs de l'école maternelle et primaire.

- Les parents sont le plus souvent favorables à cette approche, qui leur permet parfois de prolonger l'expérience scolaire à la maison. 
مجلة كلية التربية بالإسماعيلية - العدد السادس والأربعون - يناير · · P (ص VY-OV)

Jean Pierre Cuq et Isabelle Gruca distinguaient quatre types d'activités dites ludiques :

1. Les jeux linguistiques:

Ce sont tous les jeux qui se rapportent à la langue, la langue traitant des multiples composantes d'activités langagières, en particulier. «Les jeux grammaticaux, morphologiques, syntaxiques, lexicaux, phonétiques et orthographiques ».

Exemples: les mots croisés, le jeu de simulation, le jeu de plateforme, le jeu de rôles, le jeu en réseau.

2. les jeux de créativité:

Ils consistent à exploiter les connaissances langagières des apprenants d'une manière originale et inédite

Exemple: Les charades : c'est un jeu qui consiste à créer des mots en à partir des syllabes définies.

3. Les jeux culturels:

Ce genre de jeu fait appel à l'apprenant de donner d'autres connaissances des cultures.

4. Les jeux dérivés de théâtre:

Selon Jean- Piérre Cuq; Isabelle Gruca (2003), ce sont " les jeux qui transforment la salle de classe en scène théâtrale, les apprenants en acteurs, et qui reposent sur l'improvisation [...], la dramatisation, la directivité [...]"».

Donc l'apprenant est capable d'acquérir une langue et ses règles en jouant.

\section{B. L'apprentissage coopératif:}

Apprendre est un processus actif et constructif, une action menée par l'élève pour traiter l'information, l'intégrer à sa structure cognitive et élaborer des liens avec ses connaissances. De ce fait, les enseignants doivent être conscients de ces différences et choisir les méthodes et les techniques les plus convenables aux besoins des élèves.

On peut dire que l'un des moyens les plus efficaces de mener à bien dans la classe un travail coopératif qui harmoniserait ces différences, c'est de faire travailler les élèves en petits groupes. De 
cette façon, ils peuvent apprendre plus vite et mieux, et prendre plaisir à ce qu'ils étudient.

Selon Cheng et Chen (2009), l'apprentissage coopératif est considéré comme l'une des techniques d'enseignement efficaces, qui sont entrées dans le mouvement éducatif contemporain, qui a prouvé les résultats des recherches et des études sur l'éducation spécialisée et a de distinct impact positif dans la réussite scolaire des élèves et le développement de nombreuses compétences social.

Ainsi Casey et Dyson (2012), voient que l'apprentissage coopératif est considéré comme un modèle pédagogique à part entière, aussi ils voient que «il n'est pas possible d'associer l'apprentissage coopératif à un nom, à une personne, ou même à un mouvement pédagogique particulier».

Comme indiquent Vincent (2008), et Émilie Lottici (2013), Johnson et Johnson (2010), et Tardif (2009) qu'il y a plusieurs éléments qui doivent être réunis dans la position de l'apprentissage coopératif parmi lesquels.

1. L'interaction directe:

C'est-à-dire que les membres du groupe sont assis tant proche l'un de l'autre pour que tous se puissent voir et écouter sans peine.

2. La responsabilité individuelle:

Ça veut dire que chaque membre du groupe se sent responsable non seulement pour son propre processus d'apprentissage, mais aussi pour celui des autres membres du groupe et qu'il contribue à la finalisation de l'exercice demandée

3. La dépendance mutuelle positive:

$C$ 'est apparu quand tous les membres du groupe se sentent unis dans le vouloir d'atteindre un objectif commun. Pour que le groupe puisse réussir, chacun doit réussir

4. Les qualités relationnelles:

Des formes d'interaction, qui contribuent à un déroulement couronné de succès pour tous les membres du groupe (par exemple : les membres parlent un à un, s'encouragent, s'écoutent, expliquent des problèmes, interrogent l'entendement, s'aident). Ces qualités animent la communication, la confiance, les qualités de chef, les esprits de décision et le management de crise.

5. La réflexion et l' évaluation 


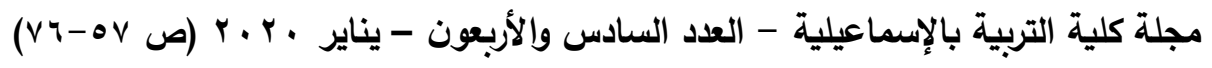

Dont les membres du groupe réfléchissent et évaluent ses efforts communs pour améliorer ses compétences coopératives et ses stratégies de travail continuellement.

Il existe toutes sortes de stratégies que les enseignants peuvent utiliser en classe. On peut citer des exemples accompagnés d'explications sur l'utilisation des stratégies de l'apprentissage coopératif:

\section{Les stratégies de l'apprentissage coopératif}

A. La stratégie Ensemble, vérifions est une stratégie qui favorise l'interaction et la collaboration entre élèves et permet à ces derniers d'apprendre à surveiller eux-mêmes leur travail. Une liste énonçant les étapes à suivre est affichée. Lorsque le travail est terminé, chaque élève vérifie que tout a été accompli.

B. La stratégie Cercle communautaire sert à créer un sentiment de solidarité et incite à l'écoute active. Tous les élèves forment un grand cercle et, à tour de rôle, partagent leurs idées et informations. Le cercle communautaire peut servir à résoudre des problèmes, à faire un remue-méninge pour un travail de rédaction ou de recherche, à donner des commentaires, etc.

C. La stratégie Co-op Co-op, les élèves sont assignés à des groupes hétérogènes dans lesquels chaque membre est responsable d'un élément d'une tâche donnée. Ils doivent faire des recherches sur un aspect du sujet à l'étude et communiquer les résultats de ce travail au groupe. Les minirapports sont ensuite incorporés dans un exposé de groupe. Vous trouverez à la page 5.10 du document Le succès à la portée de tous les apprenants une description des dix étapes à suivre pour mettre en œuvre la stratégie Co-op Co-op.

D. La stratégie Les quatre coins permet l'échange d'idées et la discussion d'idées. Affichez un sujet dans chacun des coins de la salle de classe. Les élèves choisissent celui qui les intéresse, s'installent dans le coin correspondant et se mettent par deux pour discuter du sujet en question. Ils forment ensuite un groupe de quatre et, à tour de rôle, paraphrasent ce que leur partenaire leur a dit. 
E. La stratégie Face à face s'agit d'une stratégie d'apprentissage qui encourage la discussion et l'écoute active. Le Face à face encourage la discussion au sein d'un grand groupe. Les élèves forment deux cercles : ceux du cercle intérieur font face à l'extérieur et ceux du cercle extérieur font face à l'intérieur. Posez une question. Les élèves qui se trouvent l'un en face de l'autre discutent de la question et s'entendent sur une réponse. Demandez une réponse à un groupe d'élèves. Les cercles d'élèves tournent pour avoir un nouveau partenaire. Posez une autre question.

F. La stratégie Jigsaw ou apprentissage coopératif avec décloisonnement en équipes d'experts (conçue en 1978 par Aronson, Blaney, Stephan, Silkes et Snapp) est semblable à Co-op Co-op du fait que les élèves deviennent des experts sur un aspect du sujet étudié. Tous les groupes doivent traiter le même sujet et les élèves sont répartis en groupes de base. Chacun des membres du groupe de base doit se pencher sur un sous-thème. Ils se joignent ensuite à des groupes d'experts pour travailler avec ceux qui étudient le même sous-thème. Une fois la recherche terminée, les élèves retrouvent leur groupe de base et enseignent le sous-thème aux autres membres. (Citoyenneté Éducation et al. : 2005)

Il faut signaler que le rôle de l'enseignant dans l'application dans les stratégies précèdent est central pour assurer la participation de tous les élèves. En effet, si l'enseignant laisse les élèves s'organiser comme ils le souhaitent, certains élèves vont prendre davantage de place alors que d'autres vont rester en retrait.

En fait, les enseignants qui utilisent l'apprentissage coopératif en classe voient leur rôle se diversifier. Ils ne sont plus les uniques personnes à transmettre leur savoir. Tantôt personnes-ressources, observateurs, experts, facilitateurs, les enseignants qui vivent l'apprentissage coopératif planifient et structurent leur enseignement de façon que leurs élèves puissent apprendre mutuellement tout en s'entraidant et en partageant.

Il faut, pour cela, apprendre à déléguer, à "lâcher prise", à faire confiance, tout en conservant la maîtrise de la situation. Cette méthode motivante et dynamique, qui concourt à rendre les élèves 


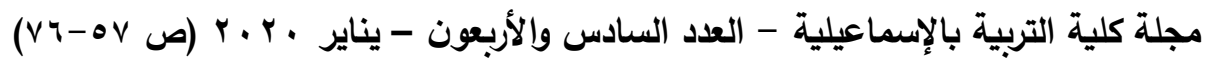

plus autonomes dans leurs apprentissages, est tellement bénéfique qu'il vaut bien la peine de s'y engager.

Selon Reverdy (2016), pour mettre en place l'apprentissage coopératif, l'enseignant doit organiser les situations de coopération selon différentes modalités qui dépendent des objectifs d'apprentissage visés : ce peut être le tutorat, où un élève est expert, l'autre novice; ou encore l'aide spontanée entre les élèves, pour pallier aux difficultés ponctuelles.

Mais la coopération évoque d'abord le travail en petits groupes autour d'une activité précise : comment choisir les élèves des groupes ? Combien en faut-il par groupe? Quelles sont les activités qui nécessitent une situation de coopération? Faut-il utiliser un système de récompense pour motiver les groupes? Autant de questions qu'aborde ce Dossier de veille, en explicitant la manière dont les recherches sur l'apprentissage en coopération peuvent nourrir les différentes pratiques existantes, et comment elles s'inspirent en même temps de ces pratiques, rendant par là-même vaine et simpliste toute tentative d'application directe des unes vers les autres.

Selon Rouiller et Howden (2010), l'enseignant doit tenir compte de certaines valeurs coopératives : tels que respect, entraide, ouverture aux autres, engagement, droit à la différence, solidarité, etc. et de celles de l'apprenant lors de la planification de situations d'apprentissage coopératif et ne pas utiliser cette approche seulement pour remplacer le travail individuel, le travail en équipe ou le travail collectif : il s'agit, au fond, d'aller au-delà du travail collaboratif en équipe, où le professeur demande par exemple aux élèves d'effectuer un exercice en équipes de deux.

Par conséquences, les défis auxquels les enseignants sont confrontés aujourd'hui sont devenus très complexes, en ce sens qu'ils comportent des dimensions variées qui s'enchevêtrent inextricablement.

sont:

Parmi ces défis, il y en a cinq particulièrement exigeants qui

- Comment entretenir la motivation des élèves?

- Comment tenir les élèves actifs et aux commandes de leur propre démarche d'apprentissage? 
- Comment faire face à l'hétérogénéité de plus en plus grande des groupes d'élèves?

- Comment favoriser un apprentissage en profondeur?

- Comment faire étudier les élèves dans un cadre qui les prépare à leur futur contexte de travail?

Pour résumer, nous proposons quelques conseils pour l'enseignant : lorsqu'il explique l'apprentissage coopératif en classe, il présente les différents rôles petit à petit en commençant par des rôles simples comme l'animateur, le secrétaire, le rapporteur et le chronométreur. À mesure que les élèves se familiarisent avec les rôles et qu'ils avancent vers des niveaux présecondaire et secondaire, vous introduirez des rôles plus compliqués.

Pour s'initier, au début, l'enseignant peut demander aux élèves de travailler en groupes sans assumer de rôles particuliers pour s'habituer à travailler ensemble. L'enseignant prévoie une rotation pour que chaque élève puisse assumer différentes responsabilités. Il doit donner au groupe un travail précis et concis ainsi qu'un objectif bien défini soit atteint. À un moment donné, l'enseignant peut proposer à ses élèves de faire une auto-évaluation.

Quant au rôle des élèves, il est consisté à s'impliquer par leur participation au travail d'équipe et à collaborer à l'amélioration de cette pratique notamment par l'utilisation d'une fiche d'autoévaluation.

Dans les salles d'enseignants, on entend souvent dire des élèves: "Ils parlent tout le temps, pas moyen de les faire taire! Ils ont toujours quelque chose à dire!" Dits parfois avec exaspération mais aussi avec humour, ces commentaires décrivent tout de même une réalité où 1 'élève affirme son besoin de communiquer. Ce besoin des enfants d'utiliser la parole semble par ailleurs être reconnu et respecté de plus en plus par ces enseignantes et enseignants.

À cette fin, le travail d'équipe apparaît comme un moyen de répondre à ce besoin impérieux des élèves de s'exprimer vocalement.

Ensergueix (2010) a identifié quatre catégories de bénéfices liés aux mises en œuvre de l'apprentissage coopératif dans un contexte scolaire traditionnel: 


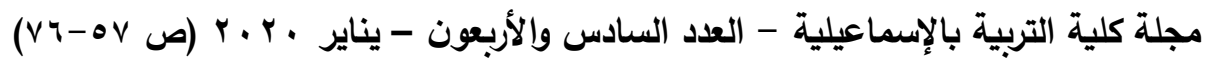

- des bénéfices scolaires: l'apprentissage coopératif participe au développement des compétences réflexives, stimule la pensée critique, améliore les compétences communicationnelles verbales, créé des atmosphères favorables à l'apprentissage exploratoire, développe la responsabilisation des élèves) ;

- des bénéfices psychologiques: l'apprentissage coopératif favorise l'acceptation plus marquées des élèves de l'aide de leurs pairs dans ce contexte pédagogique (Hertz-Lazarowitz, Kirkus, et Miller, 1992), la diminution du stress des élèves du fait de l'évitement de situations scabreuses de communication ratée face à la classe. Il incite à regarder différemment l'erreur et la demande d'aide a contrario d'un enseignement classique où celles-ci sont associées à un aveu de faiblesse (Cook et Pelfrey, 1985).

- des bénéfices évaluatifs: l'enseignant a de plus nombreuses occasions de rencontrer les élèves dans leur activité réelle, ce qui est significatif de son niveau de compétence et de compréhension, ce qui est beaucoup plus complexe en restant face à la classe. Ceci va dans le sens des propositions d'Hattie (2013 cité par Zegarac, 2013, p ; 16) qui démontre que les pratiques efficiences organisent une optimisation de la quantité de feedback indispensable pour apprendre.

- des bénéfices sociaux: développement des compétences prosociales et propices à la gestion de conflits, développement d'attitudes empathiques, développement des relations interpersonnelles, apprentissage des règles de vie en communauté, ouverture à la diversité culturelle, éprouve le concept de citoyenneté en faisant bon usage de la critique et de différents rôles sociaux (Johnson, Johnson et Holubec, 1994).

Ces résultats incitent à utiliser les méthodes d'AC pour des effets autres que les acquisitions scolaires traditionnelles. Les CS font partie de ces éléments sur lesquels, ces méthodes d'apprentissage agissent significativement comparativement à l'apprentissage individuel.

\section{Cadre pratique:}

Dans le but d'appliquer un module des activités ludiques basées sur les stratégies de l'apprentissage coopératif pour le développement des compétences langagières chez les élèves des écoles préparatoires en FLE, la chercheuse a définis les éléments suivants. 


\section{Problématique de l'étude :}

Consulter les études antérieures concernant l'évaluation des des compétences langagières et aussi ,La chercheuse a observé selon son travail comme enseignante de la langue française à l'école préparatoire Ali Moubarak que la monotonie en enseignant la langue française pour cela, elle a proposé des activités de deux, trois, quatre, cinq, six personnes, dans un module, celui-ci permet de rompre cette monotonie.

On peut déterminer la problématique de l'étude dans la question suivante:

Quelles est l'efficacité du module des activités ludiques basées sur les stratégies de l'apprentissage coopératif sur le développement des compétences langagières chez les élèves de l'école Ali Moubarak à Ismaïlia ?

D'autres questions sous-jacentes découlent de la question principale :

- Quelle sont les caractéristique d'un module des activités ludiques basées sur les stratégies de l'apprentissage coopératif pour le développement des compétences langagières chez les élèves des écoles préparatoires en FLE?

Quelle est l'efficacité de ce module proposé sur le développement compétences langagières chez les élèves de deuxième année préparatoire de l'école Ali Moubarak à Ismaïlia ?

\section{Objectif de l'étude}

Cette étude a pour but de ...

- Favoriser la prise de parole des élèves en jouant en proposant des activités ludiques basées sur les stratégies de l'apprentissage coopératif. Ces activités ludiques facilitent la mémorisation et entraînent les élèves à s'exprimer oralement et par écrit, c'est afin de développer leurs compétences langagières à l'oral et à l'écrit.

- Concevoir des activités ludiques et motivantes pour développer la performance langagière à l'oral et à l'écrit.

- Rendre les cours de français plus variés et ludiques, tout en gardant les objectifs pédagogiques. 


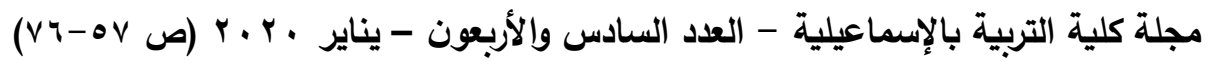

3. Importance de l'étude :

Cette étude a son importance dans le domaine de l'apprentissage/ enseignement de langue, parce qu'il est probable qu'elle puisse ...

- Favoriser l'acquisition de nouvelles compétences langagières chez les élèves.

- Soutenir et faciliter le transfert des connaissances en effectuant les objectifs des stratégies de l'apprentissage coopératif.

- Créer un contexte favorable à la discussion des connaissances en s'amusant, au sein d'un groupe de coopération,

- Améliorer la qualité de l'apprentissage en soutenant le transfert des connaissances.

\section{4. Échantillon de l'étude :}

L'échantillon se compose de 20 élèves de deuxième année à l'école préparatoire Ali Moubarak à Ismaïlia. Un seul groupe expérimental, selon le niveau des élèves, (excellent - moyen - faible). A savoir que le choix de l'échantillon dépend de l'analyse des résultats issus du pré-test élaboré par la chercheuse.

\section{Limite de l'étude :}

Cette étude s'est limitée à ...

- un échantillon aléatoire simple d'élèves de la $2^{\text {ème }}$ année à l'école préparatoire Ali Moubarak à Ismaïlia.

- La chercheuse a appliqué l'expérimentation dans le deuxième semestre de l'année universitaire (2017),

- L'application a duré deux cours par semaine, 6 cours pour l'entraînement et 6 cours d'évaluation formative, chaque cours a duré une heure, le nombre total d'heures était 12 heures pendant 12 cours.

- Le module des activités ludiques basées sur les stratégies de l'apprentissage coopératif.

\section{Outils de l'étude :}

Pour effectuer les objectifs de l'étude, la chercheuse a élaboré les outils suivants :

1. Pré-test d'évaluation structurale selon les quatre compétences langagières. 
2. Échelle d'évaluation quantitative nécessaire pour les différentes activités ludiques, une liste spécifique à chaque activité en fonction des compétences langagières sollicitées et des objectifs visés.

3. Fiche d'auto-évaluation selon les quatre compétences langagières et les stratégies de l'apprentissage coopératif.

4. Post-test d'évaluation structurale selon les quatre compétences langagières.

\section{Terminologie de l'étude:}

- Les activités ludiques:

Selon le Dictionnaire Français: Ludique signifie qui relève du jeu, qui est relatif au jeu. Quelque chose de ludique possède certaines caractéristiques du jeu. Une approche ludique sera généralement amusante, récréative ou divertissante.

Selon Jean Pierre Cuq (2003) l'activité ludique est «Une activité d'apprentissage dite ludique est guidée par des règles de jeu et pratiquée pour le plaisir qu'elle procure elle permet une communication entre apprenants (collecte, d'informations, problème à résoudre, compétition, créativité, prise de décisions, etc....), orientée vers un objectif d'apprentissage, elle permet aux apprenants d'utiliser de façon collaborative et créative l'ensemble de leurs ressources verbales et communicatives» (J. P, CUQ, 2003. 160).

Définition opérationnelle : A partir de définitions précédents, nous pouvons induire que les activités ludiques sont des activités qui se font se forment des jeux et qui permettent aux apprenants de communiquer et discuter entre eux en utilisant la langue.

\section{- L'apprentissage coopératif:}

Baudrit (2005) définit l'apprentissage coopératif comme une approche interactive et structurée qui consiste à faire travailler ensemble les apprenants de capacités et de talents différents pour atteindre un même but.

\section{Méthodologie de l'étude:}

Dans le cadre de cette étude, la chercheuse a eu recours à une méthode mixte pour analyser les données d'une manière quantitative et qualitative. 


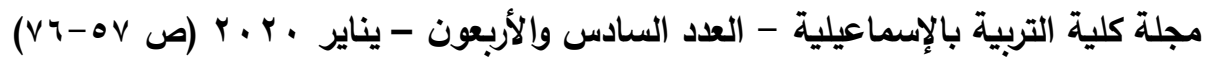

9. Procédures de l'étude:

Afin de répondre aux questions de l'étude, la chercheuse a procédé de la manière suivante:

1. Consulter la littérature et les études antérieures portant sur les variables et le sujet de l'étude:

a.Les activités ludiques.

b. Les compétences langagières.

c.Les stratégies de l'apprentissage coopératif.

2. Elaborer module proposé afin de préciser les étapes de l'application de ce module en choisissant des activités ludiques comme (Papiers pliés- Les collages - Citron-citron - Le détective - Je cherche quelqu'un qui aime - Rois et reines en rimes - Le domino ...etc.), il est à signaler que dans chaque activité, il faut préciser les points suivants: Le nom du jeu, matériel, le nombre de participants, l'âge des participants, la durée du jeu, le type de mécanismes et interactions ludiques, le but du jeu, le déroulement.

3. Préparer les outils et les matériels de l'étude pour la collecte des données :

4. Appliquer les outils sur un échantillon aléatoire simple d'élèves de la $2^{\text {ème }}$ année à l'école préparatoire Ali Moubarak à Ismaïlia.

2. Préparer une démarche de l'application de l'expérience sur le terrain.

3. Appliquer le pré-test sur les membres de l'échantillon.

4. Enseigner aux élèves de l'échantillon par le module proposé.

5. Appliquer le post-test sur les élèves de l'échantillon pour évaluer leur niveau avec compétences visées.

6. Comparer les moyennes des notes des élèves aux pré/post tests en vue de mesurer l'efficacité du module des activités ludiques basées sur les stratégies de l'apprentissage coopératif sur le développement des compétences langagières chez les élèves de l'école Ali Moubarak à Ismaiilia.

7. Présenter une interprétation quantitative et qualitative des résultats.

8. Proposer des recommandations et des suggestions à la lueur des résultats de l'étude. 


\section{Résultats de l'étude}

L'analyse des données collectées et l'interprétation des résultats de cette étude permettent de mesurer le degré de développement des compétences langagières chez les élèves de l'échantillon.

Par conséquent, l'évaluation s'avère nécessaire pour les différentes activités ludiques, on a le fait sous forme d'échelle d'évaluation spécifique à chaque activité en fonction des compétences langagières sollicitées et des objectifs visés. On a établi si, oui ou non, l'élève a accompli avec succès les actions détaillées comme suit : peut se présenter, peut décrire une personne, peut formuler une demande, comprend une consigne, respecte une consigne, peut construire une phrase complète syntaxiquement correcte, peut reproduire une structure syntaxique donnée, peut écrire sans faute d'orthographe, repère les différentes classes grammaticales, s'exprime avec aisance, maîtrise l'art de la définition. Ces compétences relèvent tant du domaine de la réception que de la production.

Afin de répondre à la question principale de l'étude "Quelles est l'efficacité du module des activités ludiques basées sur les stratégies de l'apprentissage coopératif sur le développement des compétences langagières chez les élèves de l'école Ali Moubarak à Ismaïlia ?", les données ont été décrites par le calcul de la moyenne arithmétique et de l'écart type du pré et post - test, comme il est illustré dans le tableau suivant:

Statistiques descriptives aux moyennes des notes des élèves du pré/post - test

\begin{tabular}{|c|c|c|c|c|}
\hline $\begin{array}{l}\text { Phases du module } \\
\text { proposé }\end{array}$ & Application & $\mathbf{N}$ & Moyenne & Ecart type \\
\hline \multirow{2}{*}{ 1. Prérequis } & Post-test & 20 & 15.25 & 2.82610 \\
\hline & Pré-test & 20 & 7.7 & 1.97617 \\
\hline \multirow{2}{*}{$\begin{array}{l}\text { 2. Mise en } \\
\text { application }\end{array}$} & Post-test & 20 & 15.85 & 2.34577 \\
\hline & Pré-test & 20 & 8.35 & 1.95408 \\
\hline \multirow{2}{*}{ 3. Evaluation } & Post-test & 20 & 15.7 & 2.75490 \\
\hline & Pré-test & 20 & 8.7 & 2.00263 \\
\hline \multirow{2}{*}{ Totale } & Post-test & 20 & 46.85 & 6.21776 \\
\hline & Pré-test & 20 & 24.75 & 5.42776 \\
\hline
\end{tabular}




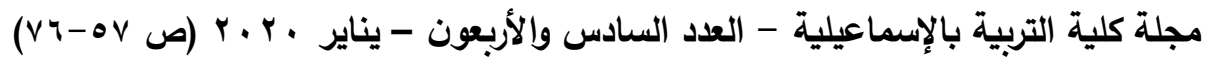

Le tableau $\mathrm{N}^{0}$ (1) indique que la valeur de la moyenne arithmétique du post-test était égale à (46.85) qui était supérieur à la moyenne arithmétique du pré-test qui était égale à (24.75) (moyenne de différence $=\mathbf{2 2 , 1}$ ).

Comme montre le tableau $\mathbf{N}^{\circ}(1)$, il y avait une augmentation dans les notes du post-test que du pré-test. Cela montre aussi l'augmentation de l'homogénéité (= Std. Ecart / Moyenne) des notes du post-test que du pré-test en raison de l'utilisation du module des activités ludiques basées sur les stratégies de l'apprentissage coopératif chez les élèves de l'échantillon. Ceci est représenté dans le graphique suivant:

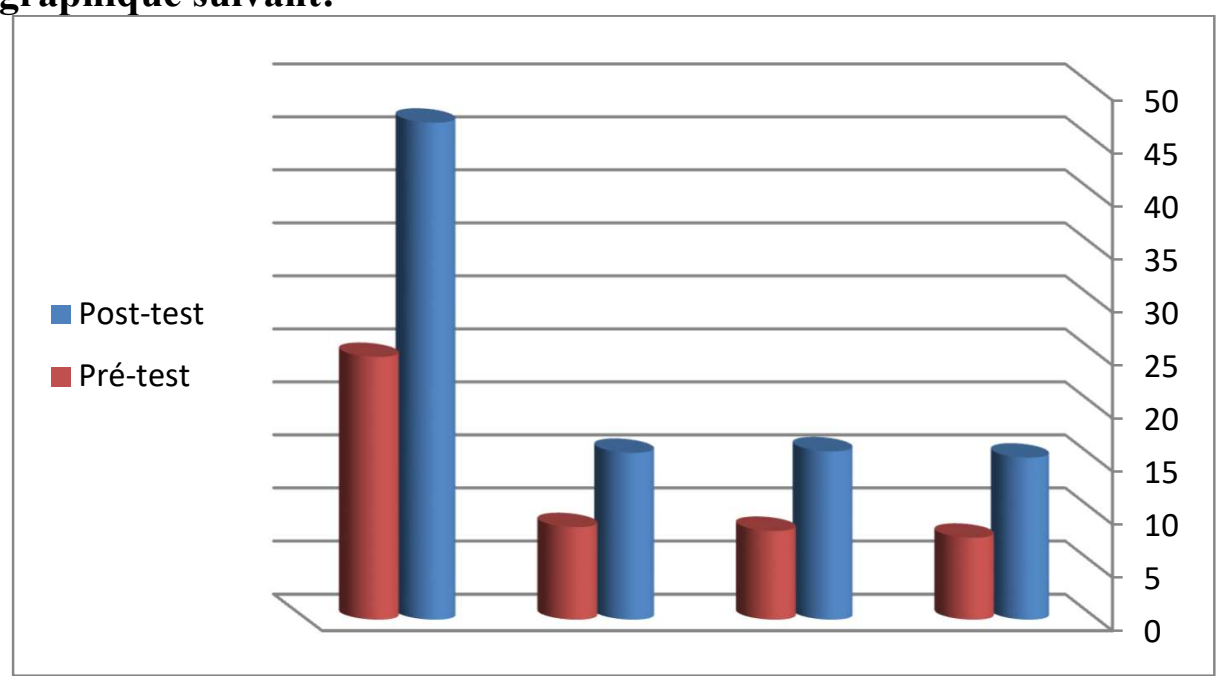

Bars représentants les moyennes des notes des élèves au pré/post - test

Ainsi, le résultat statistique de l'étude à répondre à la question de l'étude en montrant qu'" Il existe des différences statistiquement significatives entre les moyennes des notes des élèves au pré/ post-test en faveur du post teste». Cela montre qu'il y a de développements aux compétences langagières chez les élèves de l'échantillon après l'application du module proposé .

Conclusion de l'étude

Les activités ludiques font partie des moyens pédagogiques qui servent à faciliter l'apprentissage du FLE, à motiver les élèves. La majorité des enseignants de nos jours ne donnent pas importance aux jeux éducatifs qui peuvent être un moyen de motivation pour 
aimer cette langue étrangère.

Pour résumer, on peut dire que les activités ludiques ont fait l'objet d'une évaluation formative exploitable ensuite par l'enseignant pour situer le niveau de sa classe et pallier ensuite à certaines lacunes, difficultés observées lors des activités .

Le module proposé n'est qu'un bref aperçu de toutes les activités ludiques envisageables en cours de français langue étrangère qui rompent la monotonie et développent avant tout les compétences langagières d'une manière flexible chez les élèves. Il offre aussi une multitude de possibilités plus riches les unes que les autres qui sollicitent la concentration, l'écoute, la mise en pratique des diverses capacités langagières et communicatives des élèves. Enfin, on peut observer selon les résultats de l'étude que l'objectif de l'étude a été effectué. 


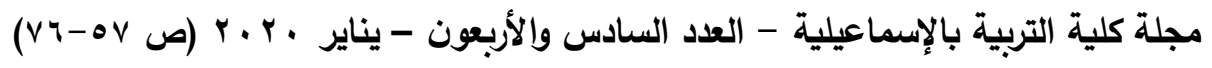

Références bibliographiques

1. BAUDRIT, A. (2005). L'Apprentissage Coopératif : origines et évolutions d'une méthode pédagogique. Bruxelles: Éditions De Bœck Université.

2. BOUDJEMAA ILHEM (2016). Le rôle des activités ludiques comme facteur de motivation dans l'enseignement du FLE (Cas des apprenants de la 2ème AM Ecole OTHMANE SAAD), Université de Larbi Tébessa - Faculté des lettres et des langues.

3. BOADIWAA MAAME AMA (2015). L'importance des activités ludiques dans l'enseignement/ apprentissage du français langue étrangère.

4. CHEN, Y \& CHENG, K. (2009). Informatique intégrant financé par l'apprentissage coopératif et résolution créative de problèmes Une stratégie d'enseignement unique. Comportement social et de la personnalité, 37 (9). 1283- 1296

5. DYSON, B. ET CASEY, A. 2012. Cooperative Learning in Physical Education. New York: Routledge.

6. ÉDUCATION, CITOYENNETE ET JEUNESSE MANITOBA. (2005). Des outils pour favoriser les apprentissages. Canada : Bibliothèque nationale du Canada EMILIE LOTTICI, (2013), Apprentissage coopératif : les représentations et pratiques des enseignants de maternelle. https://dumas.ccsd.cnrs.fr/dumas00843164/document

7. ENSERGUEIX, P. J. (2010). La formation au tutorat réciproque entre pairs pour l'acquisition d'habiletés motrices complexes. L'exemple du tennis de table au collège (Thèse de doctorat en STAPS soutenue le 29 avril 2010). Bordeaux.

8. JEAN- PIERRE CUQ \& ISABELLE GRUCA (2003). Cours de didactique de français langue étrangère seconde, Ed presse université de Grenoble.

9. JOSETTE REY -DEBOVE. (1991). Le Robert, dictionnaire de français. Paris.

10. JOHNSON, DAVID W. ET JOHNSON, R. T. (1994). Learning together and alone: cooperative, competitive, and individualistic learning. Boston: Ellyn and Bacon.

11. LAROUSSE (2006). Nouveau Dictionnaire de français.

12. REVERDY CATHERINE (2016). La coopération entre élèves : des recherches aux pratiques. Dossier de veille de l'IFÉ, $n^{\circ} 114$, décembre. Lyon : ENS de Lyon. 
13. ROUILLER, Y. et J. HOWDEN. (2010). La pédagogie coopérative : reflets de pratiques et approfondissements, Montréal, Chenelière éducation.

14. SIGMUND FREUD (2010): Principe du Plaisir et Névrose traumatique. Principe du Plaisir et Jeux d'enfants, in "Au-delà du principe de plaisir" (1920), chap. 2, Payot, coll. "Petite Bibliothèque Payot".

15. SOLTANI HABIBA (2017), L'importance des activités ludiques dans l'enseignement/ apprentissage du FLE. cas des apprenants de 3èmeannée primaire de l'école (Mahieddine Ali Ben MaamarChéria) - Tébessa.

16. VINCENT, S. (2008), 5 Eléments fondamentaux de l'apprentissage coopératif, http://90plan.ovh.net/ rotative/blog/index.php?2008/04/18/42-5elements-fondamentaux-de-l-apprentissage-cooperatif 


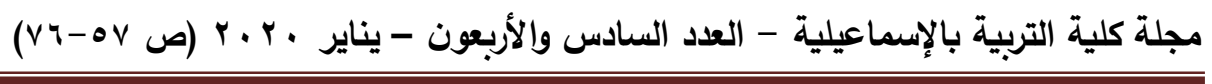

Efficacité du module des activités ludiques basées sur les stratégies de l'apprentissage coopératif pour le

développement des compétences langagières chez les élèves des écoles préparatoires en FLE

\section{RÉSUMÉ}

Dans cette étude, on tente de travailler sur un module des activités ludiques basées sur les stratégies de l'apprentissage coopératif et son importance dans le développement des compétences langagières en FLE chez les élèves de la 2ème année du cycle préparatoire à l'école Ali Moubarak à Ismaiilia.

Ainsi la chercheuse présente-t-elle, dans un premier temps le cadre théorique qui est construit principalement à partir des trois composants, le module des activités ludiques basé sur l'apprentissage coopératif et les compétences langagières. Dans un deuxième temps, une étude expérimentale : les outils et les matériels de l'étude. Dans un troisième temps, analyse et interprétation des données et des résultats de l'étude.

Mots-clés: Activités ludique - apprentissage coopératif compétences langagières.

\section{ملخص}

في هذه الدراسة، نحاول العمل على وحدة من الأنثطة الممتعة القائمة على استراتيجيات

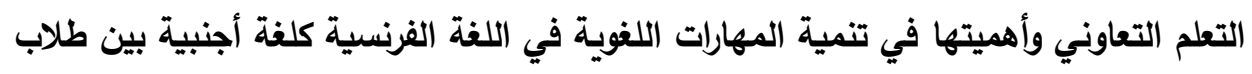

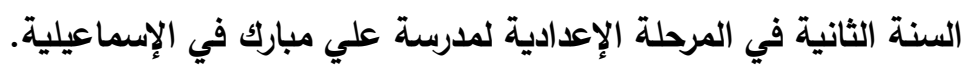

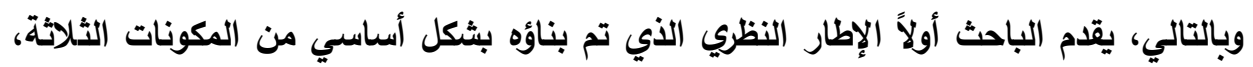

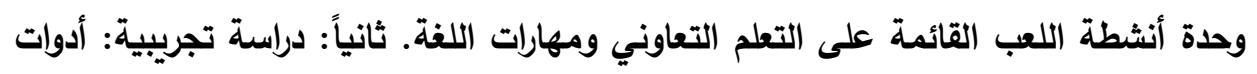
ومواد الاراسة. ثالثا: تحليل وتفسير البيانات ونتائج الدارسة. الكلمات المفتاحية: أنثطة مرحة - تعلم تعاوني - مهارات لغوية. 